\title{
High Resolution MR Imaging guidelines in retinoblastoma: prospective study correlated with histopathological results
}

\author{
Yomna S. Habib ${ }^{1 *}$ D, Ayda A. Youssef ${ }^{1}$, Hasan A. AlKiki ${ }^{2}$, Hala T. Ghareeb ${ }^{4}$ and Hossam El Dein A. ElZomor ${ }^{3}$
}

\begin{abstract}
Background: Retinoblastoma is the commonest intraocular pediatric neoplasm. With the recent scope of shifting to eye preserving therapies, preoperative MRI is becoming the primary noninvasive diagnostic method to confirm the diagnosis, assess the ocular coats and optic nerve, and detect extraocular tumor extension and intracranial primitive neuroectodermal tumor associated with retinoblastoma as funduscopy and ultrasound have limitations. Diffusion-weighted images (DWIs) detect abnormalities based on differences in tissue cellularity. Echoplanar-based (EP) DWI results in susceptibility artifacts and geometric distortions, which make DWI of the orbit a challenging technique. The purpose of our study is to evaluate preoperative magnetic resonance (MR) imaging diagnostic merits and indices in patients with retinoblastoma.
\end{abstract}

Results: Fifty-eight eyes were prospectively reviewed in 30 girls and 27 boys (mean age at diagnosis was 23 months \pm 18.9) with retinoblastoma by two radiologists on T1-weighted images, fat saturated T2-weighted images, DWIs, ADC, and postcontrast T1-weighted MR images with and without fat suppression. All patients included were primarily treated by surgical enucleation; one girl had bilateral enucleation. MRI shows accuracy, sensitivity, specificity, NPV, and PPV; $86.2 \%, 95.3 \%, 60 \%, 81.8 \%$, and $87.2 \%$ respectively for choroidal invasion; $75.8 \%, 58.8 \%$, $82.9 \%, 83 \%$, and $58.8 \%$ in prelaminar optic nerve invasion; and $86.2 \%, 85.2 \%, 87 \%, 87 \%$, and $85 \%$ in postlaminar optic nerve invasion (PLONI) respectively. For sclera and extrascleral tumor invasion, MRI show accuracy, sensitivity, specificity, NPV, and PPV of 93\%, 55.6\%, 100\%, 92.5\%, 100\%, and 98.3\%, 100\%, 98\%,100\%, 80\% respectively. On DWls, the vital solid tumor shows hyperintense signal compared to the vitreous. The difference between the mean ADC value of the solid enhancing tumor $\left(0.3[0.12-0.8] \times 10^{-3} \mathrm{~s} / \mathrm{mm}^{2}\right)$ and nonenhancing necrotic parts (1.4[1.0-1.8] $\times$ $\left.10^{-3} \mathrm{~s} / \mathrm{mm}^{2}\right)$ was statistically significant $(p<0.001)$. No correlation was found between the ADC values and high risk features for metastasis. Abnormal anterior chamber enhancement shows no statistically significant correlation with main MRI and histopathology findings. Maximum tumor diameter in $\mathrm{mm}$ show statistically significant correlation with postlaminar optic nerve invasion $(\rho=.002)$ and choroidal invasion $(\rho=.007)$. Sensitivity and specificity of different tumor size cutoff values are calculated using the ROC analysis.

Conclusion: High-resolution ocular MR imaging can reliably detect postlaminar optic nerve invasion, choroid, and extrascleral invasion; meanwhile, it shows low sensitivity in prelaminar optic nerve, ciliary body, and sclera invasion. Significantly lower ADC values in viable tumor than necrotic parts are recorded.

Keywords: MRI, Retinoblastoma, Pediatrics

\footnotetext{
* Correspondence: yomnahabib85@gmail.com

'Department of Radiology, National Cancer Institute, Cairo University, Cairo,

Egypt

Full list of author information is available at the end of the article
} 


\section{Background}

Retinoblastoma is the commonest intraocular pediatric neoplasm; its rate of incidence is 1 in 15,000 to 20,000 births worldwide. It is diagnosed in the first 5 years of life in $90-95 \%$ of patients. The most common early signs of retinoblastoma are leukocoria, a white pupil reflex, and strabismus [1]. Two forms of retinoblastoma occur; Nonheritable (sporadic) (60\%) and heritable (40\%). Sporadic retinoblastoma gene (RB1) mutations occur in a susceptible retinal cell without germline affection. Sporadic retinoblastoma is commonly solitary, unilateral; however, it can also affect both eyes (bilateral) and manifests in older age (mean age at presentation 13-18 months). All bilateral retinoblastomas have a heritable RB1 germline mutation leading to multifocal, bilateral disease and usually present earlier (mean age, 6 months) [2]. Trilateral retinoblastoma is a heritable form of retinoblastoma associated with an intracranial tumor located in the pineal, suprasellar, or parasellar regions of the brain. The incidence of trilateral retinoblastoma is $3.5 \%$ among cases with heritable retinoblastoma. Trilateral retinoblastoma is reported to be lethal, yet early diagnosis, systemic chemotherapy, and stem cell transplantation may improve the prognosis [3]. Diagnosis of retinoblastoma is usually made by funduscopy (under general anesthesia) and ultrasound, both performed by a specialized ophthalmologist. Specific tumor parameters (number, location to the macula, size of tumors, and, laterality, seeding to vitreous, subretinal space, or anterior segment) can be determined by both investigations. Ultrasound (US) is sensitive to intratumoral calcifications, the most important distinguishing feature of retinoblastoma. Doppler can be useful in differentiating a vascular intraocular mass from echogenic effusions and differentiation against pseudoretinoblastomas (e.g., persistent hyperplastic primary vitreous, PHPV). While US is adapted for evaluation of superficial ocular masses, it shows lower sensitivity in detecting deeper tumor extensions as optic nerve invasion and intracranial disease than CT and MRI. CT shows high sensitivity and specificity for the detection of calcifications. Repeated exposure to ionizing radiation and its risk of inducing cataract renders its additional diagnostic value questionable. High-resolution MRI has been considered the most sensitive diagnostic tool to evaluate intraocular masses and determine the disease extent owing to its high spatial resolution, provided the optimization of the pulse sequences and image parameters [4-6].

In the era when chemotherapy has become the main treatment for intraocular retinoblastoma, the conservative management of retinoblastoma proves to be successful in the early stages of retinoblastoma and some advanced stages avoiding the consequences of surgical enucleation and external beam radiotherapy. A new classification, the International Intraocular Retinoblastoma
Classification (IIRC), has been introduced in 2005 based on clinical examination under anesthesia. The IIRC assigns patients into groups from A through $\mathrm{E}$, according to the chemotherapy success rate. Group A, B, and C eyes show a $>90 \%$ success rate and a $47 \%$ success rate for group D eye. In eyes with high-risk histopathological features, systemic adjuvant chemotherapy is the first line of treatment in a trial to manage the eye conservatively and at the same time decrease the risk of metastasis [7]. Pathological staging had been used as the gold standard in evaluation of the high-risk factors for local recurrence and metastasis in patients undergoing enucleation. Risk factors include tumor extension into the choroid, sclera, optic nerve, and anterior eye segment. With the expanding options of nonsurgical treatment of retinoblastoma and shifting to the chemotherapeutic agents, more children have been managed without pathological staging and evaluation of metastatic risk factors [8]. Preoperative ocular MRI is becoming the primary noninvasive technique used upon deciding to treat an eye conservatively or to enucleate as funduscopy and ultrasound have limitations. MRI contributes to confirming the diagnosis, assessing the ocular coats and optic nerve to exclude high-risk features for metastasis, and detecting extraocular tumor extension and intracranial primitive neuroectodermal tumor associated with retinoblastoma (trilateral retinoblastoma) [3]. Diffusion-weighted images (DWIs) detect abnormalities based on differences in tissue cellularity. DWI acts as a supplementary noninvasive tool to characterize and differentiate benign and malignant intraocular tumors in patients presenting with leucocoria where the other main causes include includes persistent primary hyperplastic vitreous and advanced Coat's disease. DWI also differentiates viable and necrotic parts of intraocular tumor [9]. DWI can be used as a noninvasive technique to predict response to conservative therapy in retinoblastoma. DWI is based on an echoplanar sequence that is fast yet sensitive to susceptibility artifacts and image distortions, particularly at air-tissue and bone-tissue interfaces. Therefore, DWI of the orbit is challenging [10].

Although several studies have reported the diagnostic accuracy of MRI in detecting intraocular tumor extent and metastasis [3,6-15], our study provides a comprehensive evaluation of the diagnostic accuracy of $3 \mathrm{~T}$ ocular MRI, diagnostic role of DWIs, sensitivity, and specificity of different tumor diameter cut off values of postlaminar optic nerve invasion (PLONI) and ocular coat invasion as well as the relationship of the abnormal anterior eye segment enhancement in MRI examinations of retinoblastoma and different MRI and histopathological findings which, to our knowledge, are grouped in few studies. This prospective study aims to evaluate the preoperative MR imaging diagnostic value in pathologically proved retinoblastoma patients. 


\section{Methods}

\section{Study subjects}

Our prospective study was approved by our institution research department and the local ethics committee accepted reviewing the patient's records and images. Between October 2014 and January 2016, 94 patients with retinoblastoma have been referred to our tertiary care institution. Patients eligible for our study were those with pathologically proven retinoblastoma who underwent pretreatment MRI examination and primarily treated by surgical enucleation, without therapeutic interventions in the affected eye before enucleation. The inclusion criteria were met by 57 patients ( 27 boys and 30 girls, mean age at enucleation, 23 months \pm 18.9 ) (range 1-120 months); 23 patients with bilateral disease (mean age at enucleation; 17 months); and 34 patients with unilateral disease (mean age, 27 months); one patient underwent bilateral enucleation, thus data were collected from 58 eyes.

Thirty-three patients were excluded as they receive neoadjuvant chemotherapy/radiotherapy. Four patients were excluded as they have benign pathologies (Coat's disease and benign medulloepithelioma).

\section{MR technique and image analysis}

All patients were scanned on a 3T MRI machine (Ingenia 3Tesla; Philips medical systems). All were scanned under general anesthesia.

After a T1-weighted scout view of the brain, 5-mm-thick spin-echo axial T2 images of the brain were obtained. High-resolution orbital imaging was acquisied then performed: axial and coronal T1-weighted MR images were obtained with repetition time $\mathrm{msec} / \mathrm{echo}$ time $\mathrm{msec}$ of 350-530/12-14, axial and coronal T2-weighted fat saturation spin echo MR images with repetition time $\mathrm{m}$ sec/echo time $\mathrm{m}$ sec of 2250-2700/80-120 and two acquisitions. Before contrast injection, axial diffusion-weighted MRI using a spin-echo T2-weighted echoplanar imaging sequence (3200/75; section thickness, $4 \mathrm{~mm}$, with intersection gap 0.5; field of view, $230 \times 230 \mathrm{~mm}$; acquisition matrix, $190 \times 190)$. A $b$ value of 0 and $1000 \mathrm{~s} / \mathrm{mm}^{2}$ was obtained in three orthogonal planes. Apparent diffusion coefficient (ADC) maps were then constructed on the scanner.

After intravenous injection of $0.1 \mathrm{mmol} / \mathrm{kg}$ gadopentetate dimeglumine (Magnevist; Schering, Berlin, Germany), axial, sagittal, and coronal T1-weighted MR images (400-530/13-15, three acquisitions) were obtained with and without fat suppression in all patients. Axial scan covered both eyes and sagittal plane was performed in the affected eye in unilateral cases and in both eyes in bilateral cases. Finally, brain imaging was performed. Section thickness in all orbital images was $3 \mathrm{~mm}$, with an intersection gap of $0.3 \mathrm{~mm}$ for axial and $1.2 \mathrm{~mm}$ for sagittal and coronal images. A spinecho technique was used for all imaging sequences.
Two reviewers analyzed the MR images in consensus. One of the reviewers is a neuroradiology consultant who had at least 10 years' experience with brain and orbital MR imaging. Both reviewers were blinded to the patients' pathology reports. Findings were then correlated with histopathology results.

All intraocular tumors were characterized on basis of signal in both T1- and T2-weighted images as high and low relative to the vitreous. T2-weighted images and contrast-enhanced T1-weighted images were used to distinguish solid intraocular tumor from necrotic tumor where necrosis is suspected as the part of tumor of high signal intensity with corresponding nonenhancing part of the tumor. Maximum tumor diameter is measured by reviewers as the largest continuous line through the tumor mass on axial images; in the case of multiple tumor masses, the diameter of the largest mass was determined. T2-weighted MR images were used to assess retinal detachment and sub-retinal fluid. Postcontrast T1-weighted MR imaging is used to evaluate the growth pattern (endophytic, exophytic, or a combination of the two); involvement of the choroid, ciliary body, optic nerve (prelaminar, postlaminar, or no invasion), sclera, orbital fat (tumor extension), and pineal gland (trilateral retinoblastoma). The presence or absence of abnormal anterior eye segment enhancement (AASE) was evaluated. DWI were evaluated on $b=1000$ images included detection of intraocular lesions and recording of signal intensity of the lesion compared with the vitreous as high, intermediate, low. Mean ADC values were measured by placing region of interest (ROI) on enhancing tumor tissue (solid tumor part) and nonenhancing tumor tissue (areas of suspected necrosis). ROI ranges from 3 to 6 $\mathrm{mm}^{3}$.

Post contrast T1-weighted MR images of the brain are finally checked to exclude trilateral retinoblastoma or central nervous system dissemination. Histopathologic data from enucleated specimens were reported by a pathologist who was blinded to the MR imaging analysis. Tumor extension into the choroid, ciliary body, optic nerve, sclera, and orbital fat were assessed. Tumor extension into the optic nerve was categorized with reference to the lamina cribrosa of the sclera as pre- or postlaminar invasion.

\section{Statistical analysis}

Comparison of numerical variables between the study groups was done using Student $t$ test for independent samples. For comparing categorical data, Chi-square $\left(x^{2}\right)$ test was performed. Exact test was used instead when the expected frequency is less than 5. Accuracy was represented using the terms sensitivity, and specificity. Receiver operator characteristic (ROC) analysis was used to determine the optimum cut off value for the studied 
diagnostic markers. $P$ value $>0.05$ was considered insignificant. $P$ value $<0.05$ was considered significant. $P$ value $<0.01$ was considered highly significant. All statistical calculations were done using computer programs SPSS (Statistical Package for the Social Science; SPSS Inc., Chicago, IL, USA).

\section{Results}

Fifty-seven patients with retinoblastoma and primarily treated by enucleation are included in our study. Fiftythree patients had advanced disease (group D, E according to the International Classification of Intraocular Retinoblastoma), three patients (group C), and one patient (group B). Twenty-three patients (40\%) had bilateral retinoblastomas. In one patient, both eyes were enucleated. Mean age at enucleation for bilateral disease was 17 months (range, 1-48 months). In 34 patients $(60 \%)$ with unilateral retinoblastoma, right eye was enucleated in 18 patients and left eye was enucleated in 16 patients. Mean age in patients with unilateral retinoblastoma was 27 months (range, 1-120 months). Mean age at diagnosis for unilateral and bilateral cases are illustrated in Fig. 1. Histopathologic analysis was obtained in all 58 eyes. Histopathologic evaluation showed poorly differentiated tumor in $17 / 58$ eyes ( $29.3 \%$ ), necrosis $>50 \%$ in $3 / 58$ eyes $(5 \%)$, choroidal invasion in $43 / 58$ eyes (74\%), iris neovascularization in $11 / 58$ eyes (19\%), ciliary body involvement in $1 / 58$ eye (1.7\%), anterior chamber invasion in $8 / 58$ eyes (13.8\%), scleral invasion in $9 / 58$ cases $(8 \%)$, extraocular fat invasion in $4 / 58$ cases $(6.9 \%)$, optic nerve invasion in $44 / 58$ cases (75.9\%) subdivided to prelaminar optic nerve invasion occurring in 17 cases $(29.3 \%)$, and postlaminar optic nerve

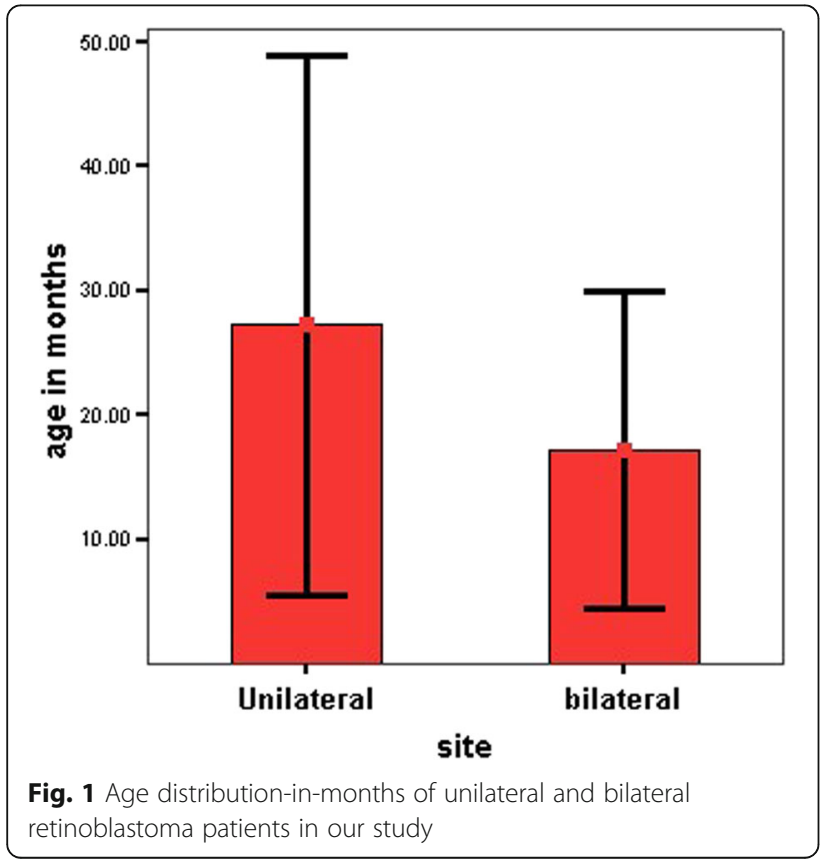

invasion in 27 cases (46.6\%). The transection line of the optic nerve was found to be involved by tumor in four eyes $(6.9 \%)$. The accuracy, sensitivity, specificity, negative predictive value (NPV), and positive predictive value (PPV) of MRI in predicting tumor invasion is summarized in Table 1. The accuracy of MRI to detect prelaminar optic nerve invasion was $75.8 \%$, with sensitivity, specificity, NPV, and PPV of 58.8\%, 82.9\%, 83\%, and 58.8\% respectively. The accuracy of MRI to detect postlaminar optic nerve invasion was $86.2 \%$, with sensitivity, specificity, NPV, and PPV of $85.2 \%, 87 \%, 87 \%$, and $85 \%$ respectively. Choroidal invasion show MRI accuracy $86.2 \%$; sensitivity, specificity, NPV, and PPV of 95.3\%, 60\%, 81.8\%, and $87.2 \%$ respectively. Sclera and extrascleral tumor invasion show accuracy, sensitivity, specificity, NPV, and PPV of 93\%, 55.6\%, 100\%, 92.5\%, 100\%, and 98.3\%, 100\%, 98\%, $100 \%, 80 \%$ respectively. Diffusion-weighted images were not feasible for interpretation in 28 eyes owing to image distortion. In 30 intraocular tumors, DWIs and ADC maps were acceptable for analysis. DWI $(b=1000)$ showed a high signal intensity of retinoblastoma compared the adjacent vitreous or sub-retinal fluid. In 3/58 intraocular tumors, heterogeneous signal intensity on DWI is seen with high signal regions of the solid enhancing tumor and intermediate signal regions of the necrotic parts with low/intermediate ADC values guided by the corresponding conventional MR images. The mean ADC value of the solid enhancing tumor was $0.3(0.12-0.8) \times$ $10^{-3} \mathrm{~s} / \mathrm{mm}^{2}$ which is significantly lower compared to nonenhancing necrotic parts with mean ADC value of $1.4(1.0-1.8) \times 10^{-3} \mathrm{~s} / \mathrm{mm}^{2}(p<0.001)$. No correlation was found between the ADC values and the degree of postlaminar optic nerve invasion, choroid invasion, sclera, and ciliary body invasion.

The association between the abnormal anterior eye segment enhancement in 35 eyes and main MRI and histopathological findings are summarized in Table 2. No statistically significant relation is concluded between anterior eye segment enhancement and tumor size $(p=$ $0.28)$, pathologically proven choroid invasion $(p=0.15)$, pathologically proven scleral invasion $(p=0.6)$, pathologically proven anterior eye segment invasion $(p=0.1)$, and iris neovascularization $(p=0.4)$. Mean tumor diameter in our study was $16.9 \mathrm{~mm}$ (range $14.50-19 \mathrm{~mm}$ ). Analysis of tumor size (maximum diameter in $\mathrm{mm}$ ) in relation to histopathology findings is shown in Table 3. The larger the tumor diameter the more incidence of postlaminar optic nerve invasion and choroid invasion which is statistically significant in our study. Mean tumor diameter in cases with pathologically proven postlaminar optic nerve invasion (PLONI) and choroidal invasion were $19 \mathrm{~mm}$ [range $13-40 \mathrm{~mm}$ ] $(p=0.002), 18$ $\mathrm{mm}$ [range $10-40 \mathrm{~mm}$ ] $(p=0.007)$ respectively. Using ROC curve obtained from our data (Figs. 2, 3, and 4), a 
Table 1 MR imaging features compared with histopathologic findings

\begin{tabular}{|c|c|c|c|c|c|c|c|c|c|c|c|}
\hline Histopathologic Finding & $\mathrm{N}$ & TP & FP & $\mathrm{TN}$ & FN & Sensitivity (\%) & Specificity (\%) & Accuracy (\%) & NPV & PPV & k \\
\hline Postlaminar ON invasion & 58 & 23 & 4 & 27 & 4 & 85.2 & 87 & 86.2 & 87.1 & 85.2 & 0.723 \\
\hline Prelaminar ON invasion & 58 & 10 & 7 & 34 & 7 & 58.8 & 82.9 & 75.8 & 82.9 & 58.8 & 0.418 \\
\hline Choroid invasion & 58 & 41 & 6 & 9 & 2 & 95.3 & 60 & 86.2 & 81.8 & 87.2 & 0.606 \\
\hline Scleral invasion & 58 & 5 & 0 & 49 & 4 & 55.6 & 100 & 93 & 92.5 & 100 & 0.679 \\
\hline Extrascleral invasion & 58 & 4 & 1 & 53 & 0 & 100 & 98 & 98.3 & 100 & 80 & 0.88 \\
\hline Ciliary body invasion & 58 & 0 & 13 & 44 & 1 & 0 & 77.2 & 77.2 & - & - & - \\
\hline
\end{tabular}

ON optic nerve, $N$ number, $T P$ true positive, FP false positive, $T N$ true negative, $F N$ false negative, $N P V$ negative predictive value, $P P V$ positive predictive value, $k$ kappa value

cut off value of $15.5 \mathrm{~mm}, 14.8 \mathrm{~mm}$ for PLONI and choroid invasion respectively yielded the highest sensitivity of $77 \%$, 83\% respectively (Table 4 ). For PLONI, the area under curve (AUC) was 0.70 (95\% CI 0.57-0.83) and the specificity was $43 \%(p=.008)$. For choroidal invasion, the AUC was $0.72(95 \%$ CI $0.57-0.87)$ and the specificity was $53 \%(p=.011)$. For cases with both choroidal invasion and PLONI, AUC was 0.73 (95\% CI 0.6-0.85) with sensitivity and specificity $63 \%$ and $58 \%$ respectively $(p=.003)$. No patients with trilateral or quadrilateral retinoblastoma or intracranial metastasis were included in this study (Figs. 5, 6, and 7).

\section{Discussion}

Retinoblastoma is the most common intraocular pediatric tumor with a high cure rate, yet of major local morbidity following enucleation due to vision loss. Recently, combined chemotherapy (systemic chemo-reduction, intraarterial, intravitreal, periocular) and focal therapies (laser photocoagulation, plaque radiotherapy, cryotherapy) have achieved high rates of local tumor control and eye preservation even in advanced-stage retinoblastoma (group D or $\mathrm{E}$ of the international intraocular classification of retinoblastoma) where enucleation is avoided in some cases in favor of intra-arterial and intravitreal chemotherapy [11]. It

Table 2 Relations between abnormal enhancement of the anterior segment of the affected eyes and the main MRI and histopathologic findings carrying risk of metastasis

\begin{tabular}{|c|c|c|c|}
\hline \multirow[t]{2}{*}{ MRI and Histopathology Finding } & \multicolumn{2}{|c|}{ Gadolinium enhancement of anterior eye segment } & \multirow[t]{2}{*}{$p$ value } \\
\hline & Abnormal & Normal & \\
\hline Tumor size in maximum axial diameter mean $\pm \mathrm{SD}(\mathrm{mm})$ & $1.75 \pm 0.53$ & $1.6 \pm 0.43$ & .281 \\
\hline \multicolumn{4}{|l|}{ Postlaminar optic nerve invasion } \\
\hline Yes & $20(74)$ & $7(26)$ & \multirow[t]{2}{*}{.059} \\
\hline No & $16(26)$ & $15(48.4)$ & \\
\hline \multicolumn{4}{|l|}{ Choroidal invasion } \\
\hline Yes & $29(67.4)$ & $14(32.6)$ & \multirow[t]{2}{*}{.153} \\
\hline No & $7(46.7)$ & $8(53.3)$ & \\
\hline \multicolumn{4}{|l|}{ Postlaminar optic nerve and Choroidal invasion } \\
\hline Yes & $17(70.8)$ & $7(29.2)$ & \multirow[t]{2}{*}{.248} \\
\hline No & $19(56)$ & $15(44)$ & \\
\hline \multicolumn{4}{|l|}{ Scleral invasion } \\
\hline Yes & $5(55.6)$ & $4(44.4)$ & \multirow[t]{2}{*}{.661} \\
\hline No & $31(63.3)$ & $18(36.7)$ & \\
\hline \multicolumn{4}{|l|}{ Anterior eye segment invasion } \\
\hline Yes & $7(87.5)$ & $5(55.6)$ & \multirow[t]{2}{*}{.110} \\
\hline No & $1(12.5)$ & $21(42)$ & \\
\hline \multicolumn{4}{|l|}{ Iris neovascularization } \\
\hline Yes & $8(72.7)$ & $3(27.3)$ & \multirow[t]{2}{*}{.418} \\
\hline No & $28(60)$ & $19(40.4)$ & \\
\hline
\end{tabular}

SD standard deviation 
Table 3 Tumor size (maximum diameter in $\mathrm{mm}$ ) in relation to histopathology findings

\begin{tabular}{llll}
\hline Finding at histopathology analysis & Prevalance $(\%)$ & Mean tumor diameter $(\mathrm{mm})$ & Range \\
\hline Postlaminar optic nerve invasion & & & $p$ value \\
No & $31(53.4)$ & 15 & $(6-20)$ \\
Yes & $27(46.6)$ & 19 & $(13-40)$ \\
Choroidal invasion & & & $(6-19)$ \\
No & $15(25.9)$ & 14 & $(10-40)$ \\
Yes & $43(74)$ & 18 & .007 \\
\hline
\end{tabular}

is important for diagnostic modalities to accurately predict the likelihood of high-risk features that carry poor prognosis in cases of advanced intraocular tumors where systemic chemotherapy is the treatment of choice on behalf of local chemotherapy. MRI at the available magnetic field strengths $(1.5$ or $3 \mathrm{~T})$ is a sensitive diagnostic modality for detecting high-risk features for metastasis that influence therapy, such as extraocular tumor extent and postlaminar optic nerve invasion [12].

In post-contrast MRI, postlaminar optic nerve invasion is identified as the extension of tumor enhancement into the optic nerve and/or abnormal optic nerve thickening $[9,13]$. According to the previously published studies, low sensitivity yet higher specificity values were concluded regarding the optic nerve invasion. In an analysis of optic nerve invasion in 64 eyes primarily enucleated for retinoblastoma, Hiasat et al. [12] reported the low sensitivity of MRI (42\%) for postlaminar optic nerve invasion with accuracy $84 \%$ and specificity $98 \%$. Similarly, Cui $\mathrm{Y}$ et al. [9] and De Jong $\mathrm{M}$ et al. [13] concluded mean sensitivities and specificities; $65-76 \%$ and $80-96 \%$ respectively for postlaminar optic nerve invasion. Sirin S. et al. [14] reported sensitivity and specificity; $75 \%$ and 95.1\% respectively for postlaminar optic nerve infiltration. Our study shows higher accuracy and sensitivity; $86.2 \%$ and $85.2 \%$ respectively for postlaminar optic nerve invasion with promising NPV (87\%). Our data show some cases with optic nerve enhancement on MR images recognized as false-positive findings. Brisse $\mathrm{HJ}$ et al. [15] assumed that false-positive results in their study might be due to bulging of the lamina cribrosa beyond its assumed site secondary to increased intraocular pressure. The prelaminar optic nerve invasion is the interruption of the continuous choroid-retinal complex linear enhancement at the optic nerve head (ONH) [13]. In our study, MRI shows low sensitivity and PPV in the prediction of prelaminar optic nerve invasion yielding accuracy, sensitivity, and specificity; 75.8\%, 60\%, and $82.9 \%$ respectively. These results are concordant with Hiasat et al. [12] who reported in their retrospective study accuracy, sensitivity, and specificity; $77 \%, 89 \%$, and 98\% respectively. Chawla et al. [16] reported sensitivity, specificity, and accuracy of $60 \%, 90 \%$, and $84 \%$ respectively. We attributed the low sensitivity in our study to a large number of tumors with microscopic invasion of the optic disc limiting the diagnostic accuracy of MRI for superficial optic nerve invasion. Fortunately, the survival rates in patients with prelaminar optic nerve invasion are $83-86 \%$ which is almost equivalent to patients with no optic nerve invasion. Whereas it is $33-55.6 \%$ for patients with postlaminar optic nerve invasion, and 19$36 \%$ for patients with optic nerve margin invasion. MRI shows higher accuracy in detecting postlaminar optic nerve invasion and is valuable in therapy planning for
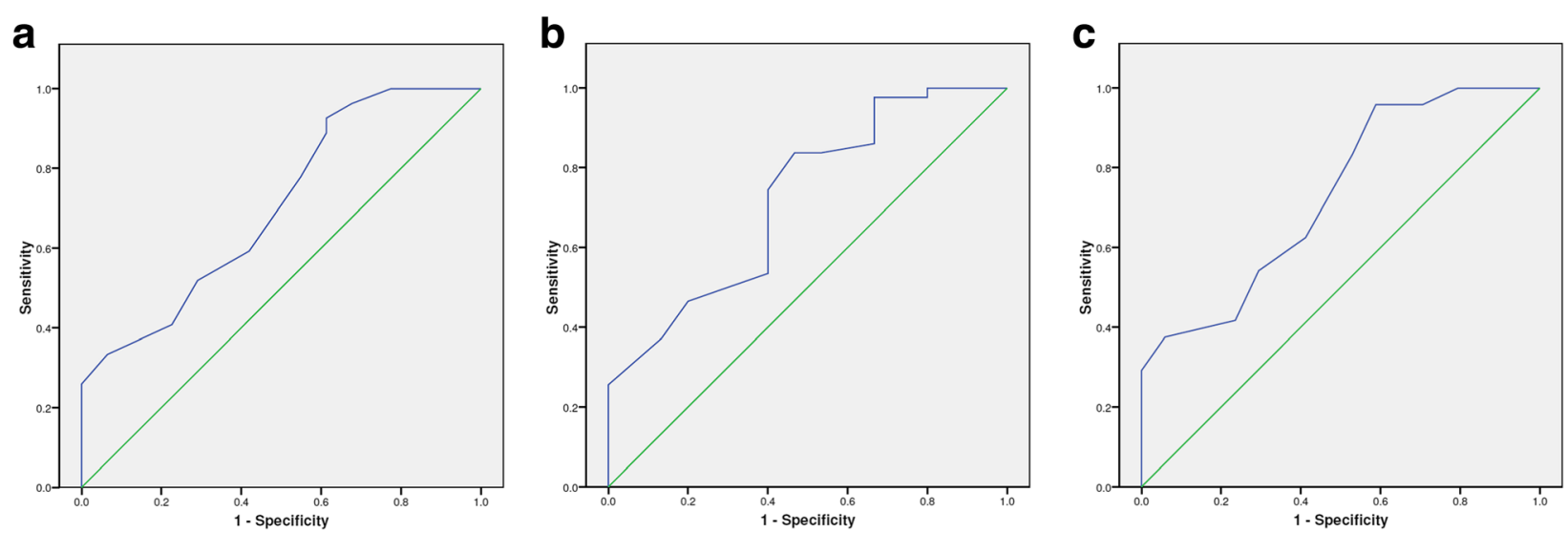

Fig. 2 ROC curves of tumor diameter in cases with postlaminar optic nerve invasion (a), choroidal invasion (b), and both postlaminar optic nerve and choroidal invasion (c) 

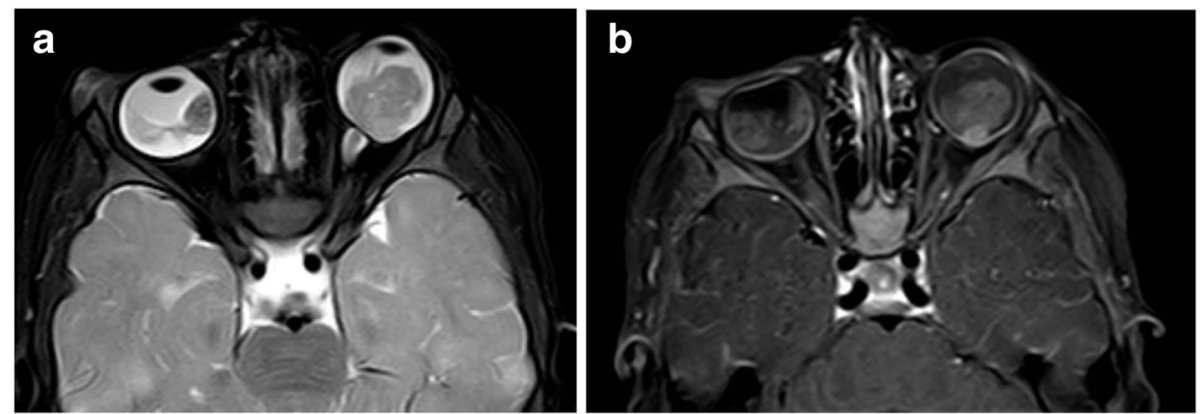

Fig. 3 Bilateral retinoblastoma, choroid invasion. Axial T2-weighted MR image (a) shows bilateral retinoblastoma, exophytic pattern with retinal detachment, and subretinal fluid. Postcontrast axial images of both orbits with fat suppression (b) show heterogeneous enhancement of the intraocular tumors. Local thickening and enhancement of the choroid adjacent to the tumor

those groups of patients at risk of metastasis. The scleral invasion is identified as interruption of the low signal sclera in T2-weighted images and extension of the tumor enhancement into the normally unenhanced sclera in postcontrast images. Extraocular fat invasion is identified as the extension of tumor enhancement in extraocular fat in fat suppression postcontrast images [13]. In our study, MRI shows accuracy for sclera invasion $93 \%$ with sensitivity and specificity; $55.6 \%$ and $100 \%$ respectively. The high rates of false-negative results were attributed to partial or microscopic trans-scleral invasion. Scleral/extra-scleral invasion by MRI shows an expected high accuracy, sensitivity, specificity, 98.3\%, $100 \%$, and $98 \%$ respectively. Cui Y. et al. [9] reported high diagnostic accuracy (98\%) for sclera/extrascleral invasion that can be excluded with high NPV. Similarly, De Jong $M$. et al. [15] reported sensitivity/specificity, $88 \% / 99 \%$ for scleral/extrascleral invasion respectively. Sirin S et al. [16] and Galluzzi P et al. [17] concluded $100 \%$ sensitivity and specificity of MRI for assessing the sclera/extrascleral invasion. The choroid is the inner highly vascular ocular layer and it lies between retina and sclera. In postcontrast T1-weighted MR images, choroid invasion is defined as an abnormal thickening of the choroid or interruption of the continuous choroidretinal complex linear enhancement by the overlying tumor [9, 13]. In our study, choroid invasion is suspected in $47 / 58$ eyes ( $81 \%), 41 / 58(70 \%)$ were truly positive, $6 / 58$ eyes were false positive, only $2 / 58$ eyes were false negative missed on MR images yielding high accuracy and sensitivity; $86.2 \%$ and $95 \%$ respectively and low specificity; $60 \%$. Low specificity might be attributed to the large mean tumor size included in our study that might hinder the delineation between the intraocular tumor and the underlying ocular wall. Our results are similar to previous reports (accuracy, 60-88\%; sensitivity, 35-82\%; and specificity, 40-99\%) [8, 12, 13]. Cui Y. et al. [9] reported an accuracy of $61.9 \%$ with very low specificity (34\%) compared to previous reports attributed to image parameter factors. One eye $(1 / 58)$ with histopathologically proven ciliary body invasion was included in our study and this finding was missed on imaging (false negative). This was attributed to the microscopic invasion of ciliary body that cannot be reliably detected
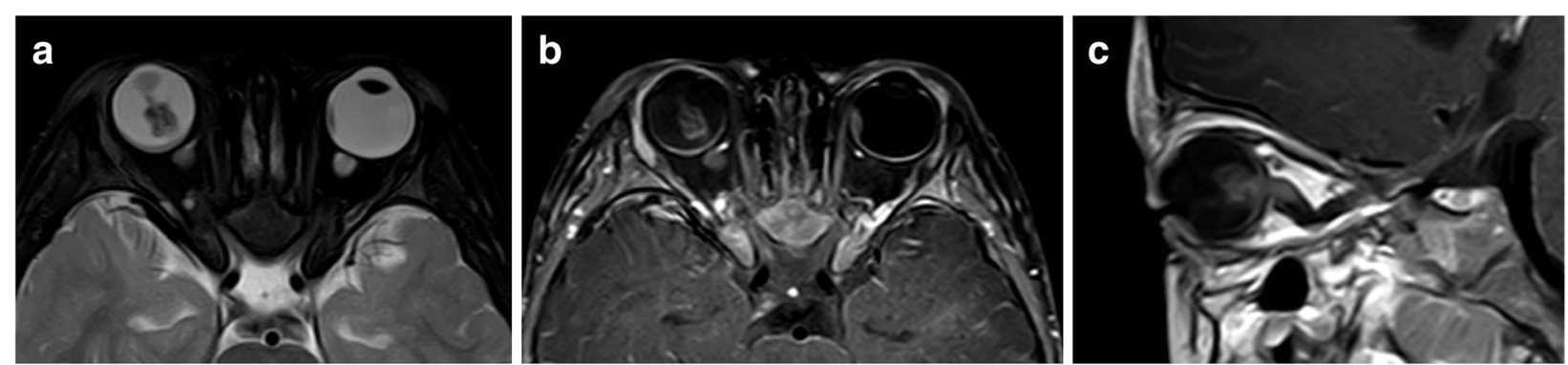

Fig. 4 Bilateral retinoblastoma and postlaminar optic nerve invasion of the right retinoblastoma. Axial T2-weighted MR image (a) shows bilateral retinoblastomas. Right retinoblastoma is exophytic with total retinal detachment and subretinal fluid and no residual vitreous. Left retinoblastoma is small focal endophytic lesion of the nasal retina, demonstrating lower signal intensity than does the ocular fluid. Postcontrast axial (b) and sagittal MR images of the right orbit (c) show bilateral enhancement of both intraocular masses. Abnormal enhancement and thickening of the optic nerve with tumor enhancement continuity in the distal optic nerve(more evident in sagittal T1 WI with contrast), a finding indicative of postlaminar optic nerve invasion. Maximum axial diameter of the right retinoblastoma $1.6 \mathrm{~cm}$. Maximum axial diameter of the left retinoblastoma $0.9 \mathrm{~cm}$ 
Table 4 Diagnostic accuracy of tumor size at different cutoff levels in the prediction of PLONI, choroid invasion, and both PLONI and choroid invasion

\begin{tabular}{llll}
\hline Parameter & Tumor size (maximum diameter in mm) & Sensitivity\% & \\
\hline Postlaminar & 15.5 & & \\
Optic nerve invasion (PLONI) & 16.5 & 59 & 53 \\
& 17.5 & 52 & 71 \\
Choroid invasion & 14.8 & 84 & 53 \\
& 15.5 & 74 & 60 \\
Both PLONI and choroid invasion & 16.5 & & 53 \\
& 17.5 & 54 & 71 \\
\hline
\end{tabular}

PLONI postlaminar optic nerve invasion

on MRI. A high rate of false-positive findings was observed (13/58), because partial-volume effects hinder differentiation between the intraocular tumor and the ciliary body. Our results were similar to previous studies [8, 12]. Cui Y. et al. [9] concluded higher specificity (98\%) and NPV than that reported in previous studies to exclude ciliary body invasion. According to our results, microscopic invasion of the prelaminar optic nerve, ciliary body, and sclera can be missed on MRI lowering its sensitivity, whereas MRI is more reliable in the detection of crucial metastatic risk factors with higher sensitivity and accuracy such as postlaminar optic nerve, choroid, and extrascleral invasion.

In our study, retinoblastoma shows restricted diffusion, demonstrating high signal on DWI at high $b$ values and low signal on ADC maps. The decrease in the ADCs in retinoblastoma is attributed to hypercellularity with enlarged nuclei and hyperchromatism resulting in a reduction in the diffusivity of water protons in the extracellular and intracellular spaces [18]. Our study included partially necrotic tumors (3/58). The region of necrosis shows less diffusion restriction and significantly higher ADC values than does the vital solid tumor. These results are in line with those reported by Razek A. et al. [18]. Cui Y et al. [9] correlated the mean ADC values of the intraocular tumor and the high-risk features for metastasis and disease dissemination. They concluded statistically significant lower ADC values with postlaminar optic nerve invasion and sclera invasion $(p=0.035$ and $p=0.07$ respectively). In our study, no statistically significant correlation between ADC values and eyes with and without high-risk features for metastasis. In our study, echoplanar (EP)-based DWIs yields susceptibility artifacts and image distortion. Hiwatashi A et al. [10] correlated EP DWIs and 3D single-shot turbo spin echo to obtain DWIs with higher spatial resolution and less image distortion. They stated that turbo spin-echo sequence is compatible to assess the diffusivity and ADC values of retinoblastoma owing to its insensitivity to field inhomogeneity despite its longer acquisition time.
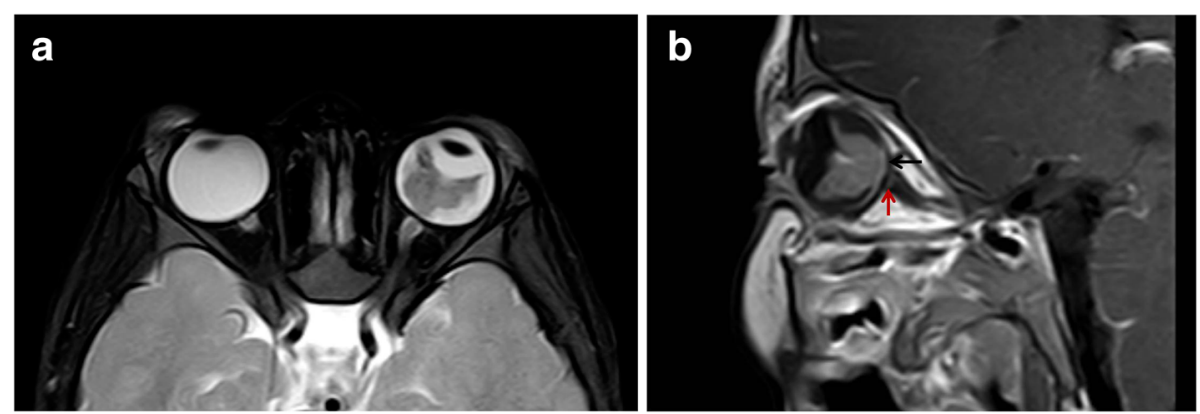

Fig. 5 Left retinoblastoma, abnormal anterior eye segment enhancement, pathology reported anterior eye segment invasion, choroid invasion, and postlaminar optic nerve invasion. Axial T2-weighted MR image (a) shows left retinoblastoma, exophytic with total retinal detachment, and subretinal fluid. Post contrast-sagittal MR images of the left orbit (b) show enhancement of the intraocular mass and abnormal anterior eye segment enhancement. Noted thickening and enhancement of the choroid adjacent to the tumor (black arrow) denoting choroid invasion. Noted the continuity of the tumor enhancement into the postlaminar optic nerve (red arrow) denoting infiltration. Maximum axial diameter $1.7 \mathrm{~cm}$ 

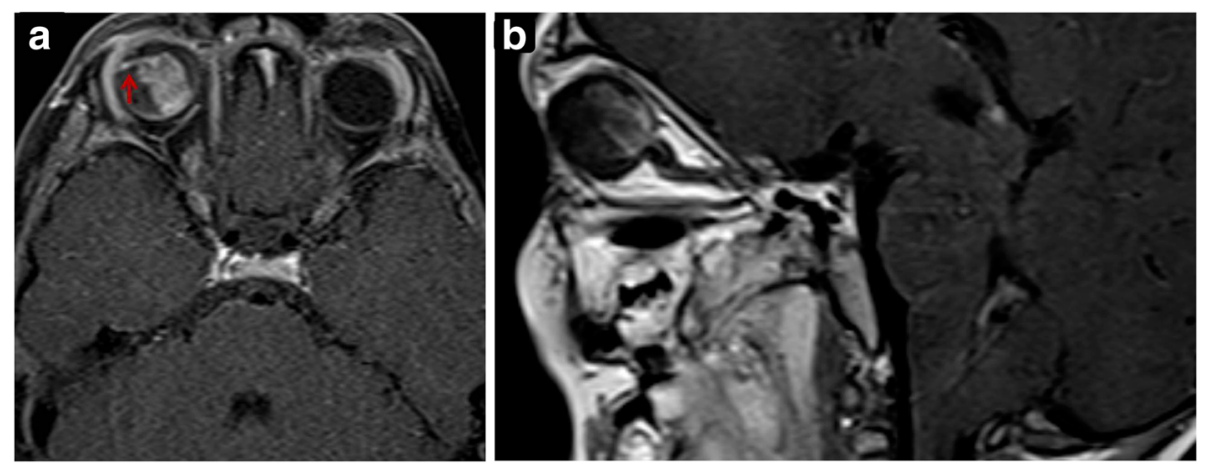

Fig. 6 Right retinoblastoma with abnormal anterior eye segment enhancement: iris neovascularization. Note postlaminar optic nerve invasion in sagittal MR image. Postcontrast axial MR image of both orbits with fat suppression (a) and sagittal MR image of right orbit (b) shows

heterogeneous enhancement of the right retinoblastoma and extreme enhancement of the iris of the right eye compared to that of the left eye ( red arrow). Note the abnormal enhancement of the distal optic nerve (sagittal MR images of the right orbit) indicative of postlaminar optic

nerve invasion. Maximum axial diameter of right retinoblastoma $1.9 \mathrm{~cm}$

In this study, the relation between the tumor size (measured as the maximum diameter in $\mathrm{mm}$ ) and postlaminar optic nerve invasion (PLONI)/choroid invasion was statistically significant; the larger the tumor size, the higher the incidence of PLONI and choroid invasion. The mean tumor diameter associated with PLONI and choroid invasion in our study was 19 and $18 \mathrm{~mm}(p=$ .002 and .007) respectively. In their studies, De Jong M. and colleagues $[6,11]$ reported that there is a statistically significant correlation between PLONI/choroidal invasion and the intraocular tumor size in patients with retinoblastoma (tumor volume in $\mathrm{mm}^{3}$ and tumor diameter in $\mathrm{mm}$ ). Mean tumor volume and diameter with PLONI were $1.70 \mathrm{~cm}^{3}$ and $18 \mathrm{~mm}$ respectively $(p \leq .0001)$. Mean tumor volume and diameter with choroid invasion were $1.4 \mathrm{~cm}^{3}$ and $16.5 \mathrm{~mm}(p=.0002)$. In their studies, Brisse $\mathrm{HJ}$ et al. [15] and Yan J et al. [17] also concluded a significant correlation between the maximal tumor diameter and incidence of PLONI. Brisse $\mathrm{HJ}$ et al. [15] reported a mean tumor diameter of $17.6 \mathrm{~mm}(p<0.001)$ with PLONI. Using ROC curve data analysis, the intraocular tumor diameter cut off values in eyes with PLONI and choroid invasion were $15.5 \mathrm{~mm}$ and $14.8 \mathrm{~mm}$, yielding a sensitivity; $77 \%$ and $84 \%$ respectively. The aim is to predict the likelihood low or high risk of incidence of PLONI or choroidal invasion aiding to implement the proper management. De Jong and colleagues [6] designed a clinically applicable tumor size cut off levels owing to the large sample size of their study.

In our study, abnormal anterior eye segment (AES) enhancement was observed in postcontrast $\mathrm{T} 1$ fat suppression images of $35 / 58$ eyes (60\%) with retinoblastoma. De Graaf and colleagues [19] reported in their univariable analysis that the degree of AES enhancement correlated significantly with histopathologically proven iris neoangiogenesis $(p=.06)$, histopathologically proven postlaminar
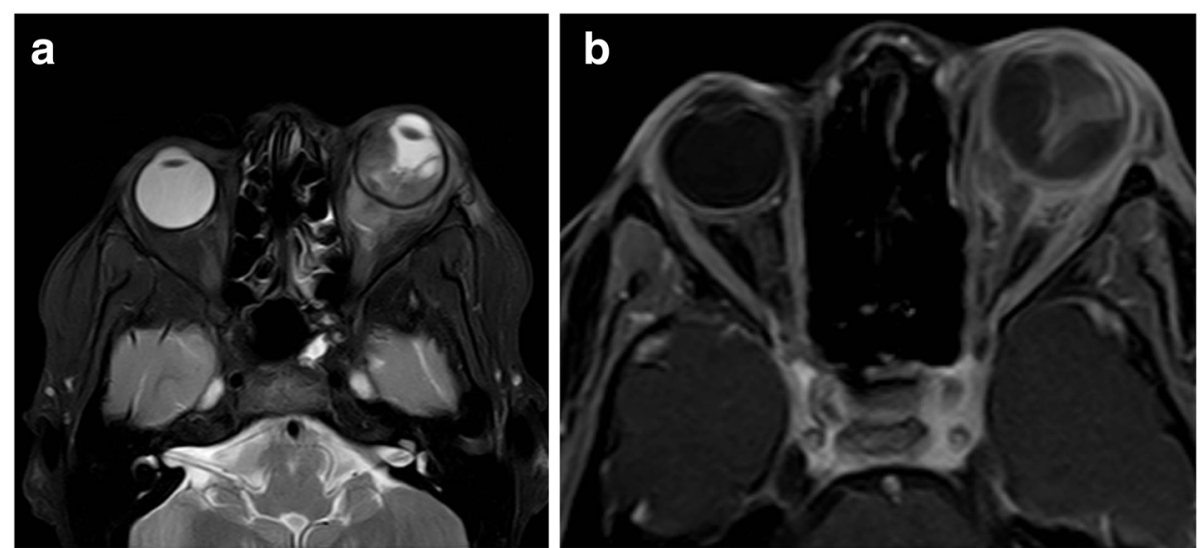

Fig. 7 Left retinoblastoma with scleral and extraocular fat invasion. Axial fat saturated T2-weighted MR image (a) shows left ocular mass with scleral invasion and extraocular fat involvement. Post contrast axial MR image with fat suppression (b) show heterogenous enhancement of left retinoblastoma due to necrosis and abnormal enhancement of extraocular fat denoting invasion. Maximum axial diameter $2.5 \mathrm{~cm}$ 
optic nerve invasion (PLONI), and large tumor size ( $p=$ $.002)$. No statistically significant association was found between AES enhancement and pathologically proven anterior eye segment invasion, choroidal and scleral invasion. In our study, no statistically significant association between abnormal enhancement of the anterior eye segment and most of MRI and histopathological variables as iris neoangiogenesis $(p=.418)$, anterior chamber tumor invasion $(p=.110)$, choroid and sclera invasion $(p=0.15$ and $p=0.66$ respectively), as well as larger tumors ( $p=$ .281). In our study, one pathological variable (PLONI) correlated with abnormal AES enhancement $(p=0.05)$.

In our study, the mean ages of children with bilateral and unilateral retinoblastoma were 17 months and 27 months respectively. Bilateral retinoblastoma shows earlier presentation according to our study which is statistically significant $(p=0.015)$. Cui Y et al. [9] in their study reported that the mean age of children with retinoblastoma was 24 months \pm 18.93 and was significantly higher in unilateral cases compared with bilateral cases $(p=0.044)$.

Our prospective study had some limitations. First, we had a relatively small sample size. Second, correlation with clinical examination was not a part of our study. Further studies should correlate MRI and ophthalmoscopy findings. Orbital DWI using echo-planer sequences is prone to susceptibility artifacts and image distortion. Further studies should apply the single-shot turbo spinecho sequences to improve the image quality.

\section{Conclusion}

High-resolution ocular MRI is the primary diagnostic tool for pretreatment assessment of retinoblastoma and acts as a substitute to pathological staging in non-surgical management of patients with retinoblastoma. Our data show higher sensitivity, accuracy, and negative predictive values in detecting the crucial high-risk factors for metastasis than that published in the literature. MRI can reliably detect postlaminar optic nerve invasion (PLONI), choroidal invasion, and extrascleral invasion with a high rate of correspondence to the histopathological data. MRI shows some limitations in detecting early optic nerve invasion, ciliary body invasion, and early/microscopic sclera infiltration in eyes with retinoblastoma. Mean ADC values are significantly lower in solid enhancing tumor than necrotic nonenhancing parts. There is a directly proportional relationship between the increase in intraocular tumor size and risk of PLONI and to lesser extent choroidal invasion. Abnormal anterior eye segment enhancement (AASE) might be a sign of increased tumor aggressiveness.

\section{Main points}

- Preoperative high-resolution brain and orbit MRI shows high sensitivity, accuracy, and negative predictive values in detecting crucial high-risk factors for metastasis in advanced stages retinoblastomas such as postlaminar optic nerve invasion and choroidal invasion. This might aid in staging especially in the scope of shifting to eye sparing therapies.

- The larger the intraocular tumor, the higher likelihood of PLONI and choroid invasion which is statistically significant in our study. A cut off value of $15.5 \mathrm{~mm}$ and $14.8 \mathrm{~mm}$ for PLONI and choroid invasion respectively yielded the highest sensitivity using ROC curve.

- DWIs echo-planar sequences are prone to susceptibility artifacts and image distortion. Further studies should apply the single-shot turbo spin-echo sequences to improve the image quality.

\section{Abbreviations}

AASE: Abnormal anterior eye segment enhancement; ADC: Apparent diffusion coefficient; AUC: Area under curve; Cl: Confidence interval; DWI: Diffusion-weighted images; EBR: External beam radiation;

EP: Echoplanar-based DWI; IAC: Intraarterial chemotherapy; IIRC: International Intraocular Retinoblastoma Classification; IVC: Intravenous chemotherapy; IVitC: Intravitreal chemotherapy; NPV: Negative predictive value; ONH: Optic nerve head; PHPV: Persistent hyperplastic primary vitreous;

PLONI: Postlaminar optic nerve invasion; POC: Periocular chemotherapy; PPV: Positive predictive value; RB1: Retinoblastoma gene; ROC: Receiver operator characteristic analysis; ROI: Region of interest; $\mathrm{T}$ : Tesla;

US: Ultrasound

\section{Acknowledgments}

This research was carried out at Children's Cancer Hospital Foundation which is a specialized (tertiary care) medical institute in diagnosis and management of neoplastic disease of children. We want to thanks our Professors and colleagues who helped us to do such research work.

\section{Authors' contributions}

YH collected patient data, patient's images and analysis and wrote the manuscript and is responsible for correspondence to journal. AY supervised and participated in the design of the manuscript. $\mathrm{HZ}$ and $\mathrm{HT}$ participated in the design of the study and performed the statistical analysis. HK conceived of the study, and participated in its design and coordination and helped to draft the manuscript. All authors read and approved the final manuscript.

\section{Funding}

No funding sources

\section{Availability of data and materials}

The datasets used and analyzed during the current study are available from the corresponding author on reasonable request.

\section{Ethics approval and consent to participate}

The study was approved by the ethical committee of "Children's Cancer Foundation of Egypt" with ethical committee approval number R-14-08-52 and approval date $8 / 2014$. An informed written consent was taken from all subjects.

\section{Consent for publication}

All patients included in this research gave written informed consent to publish the data contained within this study.

\section{Competing interests}

The authors declare that they have no competing interests.

\section{Author details}

'Department of Radiology, National Cancer Institute, Cairo University, Cairo, Egypt. ${ }^{2}$ Department of Radiology, Faculty of Medicine, Cairo University, Cairo, Egypt. ${ }^{3}$ Department of Pediatric Oncology, National Cancer Institute, Cairo 
University, Cairo, Egypt. ${ }^{4}$ Department of Pathology, National Cancer Institute, Cairo University, Cairo, Egypt.

Received: 29 November 2019 Accepted: 21 January 2020

Published online: 06 February 2020

\section{References}

1. Ortiz MV, Dunkel IJ (2016) Retinoblastoma. J Child Neurol 31(2):227-236 https://doi.org/10.1177\%2F0883073815587943

2. Feygin T, Leahey AM (2015) Retinoblastoma: Advanced Imaging, Clinical Approach. Journal of Pediatric Neuroradiology 4:82-96. https://doi.org/10. 1055/s-0036-158413

3. De Jong $M C$, de Graaf $P$, Brisse HJ, Galluzzi $P$, Göricke SL, Moll AC, Munier FL, Popovic MB, Moulin AP, Binaghi S, Castelijns JA (2015) The potential of 3T high-resolution magnetic resonance imaging for diagnosis, staging, and follow-up of retinoblastoma. Surv Ophthalmol 60(4):346-355 https://doi.org/ 10.1016/j.survophthal.2015.01.002

4. $\quad$ Shields CL, Lally SE. Retinoblastoma (2019) In Ocular Oncology pp. 91-99. Springer, Singapore

5. Marr BP, Singh AD (2015) Retinoblastoma: evaluation and diagnosis. In Clinical Ophthalmic Oncology pp. 1-11. Springer, Berlin, Heidelberg.

6. De Jong MC, van der Meer FJ, Göricke SL, Brisse HJ, Galluzzi P, Maeder P Sirin S, De Francesco S, Sastre-Garau X, Metz KA, Cerase A (2015) Diagnostic accuracy of intraocular tumor size measured with MR imaging in the prediction of postlaminar optic nerve invasion and massive choroidal invasion of retinoblastoma. Radiology 279(3):817-826 https://doi.org/10. 1148/radiol.2015151213

7. Fabian ID, Stacey AW, Chowdhury T, Duncan C, Karaa EK, Scheimberg I, Reddy MA, Sagoo MS (2017) High-risk histopathology features in primary and secondary enucleated international intraocular retinoblastoma classification group D eyes. Ophthalmology 124(6):851-858 https://doi.org/ 10.1016/j.ophtha.2017.01.048

8. Galluzzi P, Hadjistilianou T, Cerase A et al (2015) MRI Helps Depict Clinically Undetectable Risk Factors in Advanced Stage Retinoblastomas. Neuroradiol J 28(1):53-61 https://dx.doi.org/10.15274\%2FNRJ-2014-10103

9. Cui Y, Luo R, Wang R, Liu H, Zhang C, Zhang Z, Wang D (2018) Correlation between conventional MR imaging combined with diffusion-weighted imaging and histopathologic findings in eyes primarily enucleated for advanced retinoblastoma: a retrospective study. Eur Radiol 28(2):620-629 https://doi.org/10.1007/s00330-017-4993-7

10. Hiwatashi A, Togao O, Yamashita K, Kikuchi K, Yoshikawa H, Obara M, Honda H (2016) 3D turbo field echo with diffusion-sensitized driven-equilibrium preparation technique (DSDE-TFE) versus echo planar imaging in evaluation of diffusivity of retinoblastoma. Br J Radiol 89(1067):20160074 https://doi. org/10.1259/bjr.20160074

11. De Jong MC, de Graaf P, Pouwels PJ, Beenakker JW, Jansen RW, Geurts JJ, Moll AC, Castelijns JA, van der Valk P, van der Weerd L (2018) 9.4 T and 17.6 T MRI of Retinoblastoma: Ex Vivo evaluation of microstructural anatomy and disease extent compared with histopathology. J Magn Reson Imaging 47(6): 1487-1497 https://doi.org/10.1002/jmri.25913

12. Hiasat JG, Saleh A, Al-Hussaini M, Al Nawaiseh I, Mehyar M, Qandeel M, Mohammad M, Deebajah R, Sultan I, Jaradat I, Mansour A (2019) The predictive value of magnetic resonance imaging of retinoblastoma for the likelihood of high-risk pathologic features. Eur J Ophthalmol 29(2):262-268 https://doi.org/10.1177\%2F1120672118781200

13. De Jong MC, de Graaf P, Noij DP, Göricke S, Maeder P, Galluzzi P, Brisse HJ, Moll AC, Castelijns JA (2014) European Retinoblastoma Imaging Collaboration. Diagnostic performance of magnetic resonance imaging and computed tomography for advanced retinoblastoma: a systematic review and meta-analysis. Ophthalmology 121(5):1109-1118 https://doi.org/10. 1016/j.ophtha.2013.11.021

14. Sirin S, Schlamann M, Metz K et al (2015) High-resolution MRI using orbit surface coils for the evaluation of metastatic risk factors in 143 children with retinoblastoma. Part 2: new vs. old imaging concept. Neuroradiology 57: 815-824. https://doi.org/10.1007/s00234-015-1544-2

15. Brisse HJ, Graaf $P$, de Galluzzi $P$ et al (2015) Assessment of early stage optic nerve invasion in retinoblastoma using high resolution 1.5 Tesla MRI with surface coils: a multicentre, prospective accuracy study with histopathological correlation. Eur Radiol 25:1443-1452 https://doi.org/10. 1007/s00330-014-3514-1
16. Chawla B, Sharma S, Sen S et al (2012) Correlation between clinical features, magnetic resonance imaging and histopathologic findings in retinoblastoma: a prospective study. Ophthalmology 119:850-856 https:// doi.org/10.1016/j.ophtha.2011.09.037

17. Yan J, Zhang H, Li Y (2013) Establishment of the relationship between tumor size and range of histological involvement to evaluate the rationality of current retinoblastoma management. PLoS One 8(11):e80484 https://doi. org/10.1371/journal.pone.0080484

18. Razek AA, Elkhamary S, Al-Mesfer S, Alkatan HM (2012) Correlation of apparent diffusion coefficient at $3 \mathrm{~T}$ with prognostic parameters of retinoblastoma. Am J Neuroradiol 33(5):944-948 https://doi.org/10. 3174/ajnr.A2892

19. De Graaf P, van der Valk P, Moll AC et al (2010) Contrast-enhancement of the anterior eye segment in patients with retinoblastoma: correlation between clinical, MR imaging, and histopathologic findings. AJNR Am J Neuroradiol 31:237-245 https://doi.org/10.3174/ajnr.A1825

\section{Publisher's Note}

Springer Nature remains neutral with regard to jurisdictional claims in published maps and institutional affiliations.

\section{Submit your manuscript to a SpringerOpen ${ }^{\circ}$ journal and benefit from:}

- Convenient online submission

- Rigorous peer review

- Open access: articles freely available online

- High visibility within the field

- Retaining the copyright to your article

Submit your next manuscript at $>$ springeropen.com 